\title{
PET/CT Imaging of Head-and-Neck and Pancreatic Cancer in Humans by Targeting the "Cancer Integrin" av $\beta 6$ with Ga-68-Trivehexin
}

Neil Gerard Quigley

TUM: Technische Universitat Munchen

Katja Steiger

TUM: Technische Universitat Munchen

Sebastian Hoberück

Universitatsklinikum Carl Gustav Carus

Norbert Czech

Center for Nuclear Medicine and PET/CT Bremen

Maximilian Alexander Zierke

TUM: Technische Universitat Munchen

Susanne Kossatz

TUM: Technische Universitat Munchen

Marc Pretze

Universitatsklinikum Carl Gustav Carus

Frauke Richter

TUM: Technische Universitat Munchen

Wilko Weichert

TUM: Technische Universitat Munchen

Christian Pox

Krankenhaus Sankt Joseph Stift Bremen

Jörg Kotzerke

Universitatsklinikum Carl Gustav Carus

Johannes Notni ( $\nabla$ ukessen@notni.de)

Clinic for Nuclear Medicine, University Hospital Essen https://orcid.org/0000-0002-3964-3391

\section{Research Article}

Keywords: av $\beta 6$, imaging, uptake, integrin, trivehexin, cells, human, hnscc, pdac, probe

Posted Date: July 21st, 2021

DOI: https://doi.org/10.21203/rs.3.rs-719213/v1 
License: (c) (i) This work is licensed under a Creative Commons Attribution 4.0 International License. Read Full License

Version of Record: A version of this preprint was published at European Journal of Nuclear Medicine and Molecular Imaging on September 24th, 2021. See the published version at https://doi.org/10.1007/s00259-021-05559-x. 


\section{PET/CT Imaging of Head-and-Neck and Pancreatic Cancer in Humans by Targeting the "Cancer Integrin" av $\beta 6$ with Ga-68-Trivehexin}

Neil Gerard Quigley ${ }^{1 \#}$, Katja Steiger ${ }^{1 \#}$, Sebastian Hoberück ${ }^{2}$, Norbert Czech $^{3}$, Maximilian Alexander

Zierke $^{1}$, Susanne Kossatz ${ }^{4}$, Marc Pretze ${ }^{2}$, Frauke Richter ${ }^{1}$, Wilko Weichert ${ }^{1}$, Christian Pox ${ }^{5}$, Jörg Kotzerke $^{2}$, Johannes Notni*1,6

1 Institute of Pathology, Technische Universität München, Munich, Germany.

2 Klinik und Poliklinik für Nuklearmedizin, Universitätsklinikum Carl Gustav Carus an der Technischen Universität Dresden, Dresden, Germany

3 Center of Nuclear Medicine and PET/CT Bremen, Bremen, Germany.

4 Department of Nuclear Medicine, University Hospital Klinikum rechts der Isar, and Central Institute for Translational Cancer Research (TranslaTUM), Technical University of Munich, Munich, Germany

5 Clinic of Internal Medicine, Hospital St. Joseph-Stift Bremen, Bremen, Germany

6 Experimental Radiopharmacy, Clinic for Nuclear Medicine, University Hospital Essen, Hufelandstr. 55, 45147 Essen, Germany

\# These authors contributed equally.

Corresponding author: Prof. Dr. Johannes Notni

Experimental Radiopharmacy

Clinic for Nuclear Medicine

University Hospital Essen

Hufelandstr. 55

45147 Essen

Germany

Tel: +492017232032

Fax: +49 2017235964

email: johannes.notni@uk-essen.de

Word Count: 5554

Running Title: Ga-68-Trivehexin for $\alpha v \beta 6$-Integrin Targeted Cancer Imaging

Keywords: Positron emission tomography, Carcinoma, Integrins, Gallium-68 


\begin{abstract}
Purpose

To develop a new probe for the $\alpha v \beta 6$-integrin and assess its potential for PET imaging of carcinomas.

\section{Methods}

Ga-68-Trivehexin was synthesized by trimerization of an optimized $\alpha v \beta 6$-integrin selective cyclic nonapeptide on the TRAP chelator core and automated labeling with Ga-68. The tracer was characterized by ELISA for activities towards integrin subtypes $\alpha v \beta 6, \alpha v \beta 8, \alpha v \beta 3$, and $\alpha 5 \beta 1$, as well as by cell binding assays on H2009 ( $\alpha v \beta 6$-positive) and MDA-MB-231 ( $\alpha v \beta 6$-negative) cells. SCID mice bearing subcutaneous xenografts of the same cell lines were used for dynamic $(90 \mathrm{~min}$ ) and static (75 min p.i.) $\mu$ PET imaging, as well as for biodistribution (90 min p.i.). Structure-activity-relationships were established by comparison with the predecessor compound Ga-68-TRAP(AvB6)3. Ga-68Trivehexin was tested for in-human PET/CT imaging of HNSCC, parotideal adenocarcinoma, and PDAC.
\end{abstract}

\title{
Results
}

Ga-68-Trivehexin showed a high $\alpha \mathrm{v} \beta 6$-integrin affinity $\left(\mathrm{IC}_{50}=0.033 \mathrm{nM}\right)$, selectivity over other subtypes ( $\mathrm{IC}_{50}$-based factors: $\left.\alpha \mathrm{v} \beta 8,188 ; \alpha \mathrm{v} \beta 3,82 ; \alpha 5 \beta 1,667\right)$, blockable uptake in $\mathrm{H} 2009$ cells, and negligible uptake in MDA-MB-231 cells. Biodistribution and preclinical PET imaging confirmed a high target-specific uptake in tumor and a low non-specific uptake in other organs and tissues except the excretory organs (kidneys and urinary bladder). Preclinical PET corresponded well to in-human results, showing high and persistent uptake in metastatic PDAC and HNSCC ( $\left.\mathrm{SUV}_{\max }=10-13\right)$ as well as in kidneys/urine. Ga-68-Trivehexin enabled PET/CT imaging of small PDAC metastases and showed high uptake in HNSCC but not in tumor-associated inflammation.

\section{Conclusions}

Ga-68-Trivehexin is a valuable probe for imaging of $\alpha \mathrm{v} \beta 6$-integrin expression in human cancers. 


\section{Introduction}

Cancer possesses a uniquely terrifying quality in the perception of the general public - barely understood, frequently being personificated, used as a metaphor, or even mistaken as a personal trait [1]. Although other devastating pandemics have occasionally taken the lead in public agitation and fear (e.g., AIDS and recently COVID-19), cancer may still be seen as "The Emperor of All Maladies" [2], for its incidence rate in a globally ageing population that naturally grows. Already Galen reserved the metaphorical term 'cancer' to the malignant and deadly forms of solid neoplasia. Malignancy, in turn, is tantamount to infiltrative growth, metastasis, and dedifferentiation since Virchow's time [3]. Among the molecular factors governing malignancy, integrins, a class of 24 transmembrane cell adhesion receptors which are each formed by dimerization from $18 \alpha$ - and $8 \beta$-subunits, are not always the first that come to mind. Although awareness is mostly limited to the subtype $\alpha v \beta 3$ because of its well-established role as a promoter of (tumoral) angiogenesis [4,5], many other integrins are involved in every step of cancer development [6]. The dimer $\alpha \mathrm{v} \beta 6$, which is solely expressed by epithelial cells, is arguably most intertwined with carcinogenesis [7]. It drives at least two acquired capabilities of tumor cells as defined by the hallmarks of cancer [8], notably, insensitivity to anti-growth signals, and additionally, invasiveness and metastasis. The signature functionality of $\alpha \mathrm{v} \beta 6$-integrin is the activation of transforming growth factors beta 1 and 3 (TGF- $\beta$ 's) [9], which are secreted by virtually all mammalian cell types into the extracellular space in the form of an inactive complex with latency associated peptide (LAP) [10]. $\alpha v \beta 6$-integrin binds to an Arg-Gly-Asp (RGD) sequence of LAP and, by exerting a pulling force along the longitudinal axis of the $\beta 6$ protein [11], distorts the conformation of LAP and thereby ultimately releases TGF- $\beta$ [12]. The link to tumor invasion is given by the fact that tumor cells frequently develop a resistance against the overall growth-inhibiting effect of TGF- $\beta$ [13] by a loss of certain downstream signaling components, e.g., p53 [14] or Smad4 [15]. Such tumor cells can benefit from generating a high TGF- $\beta$ concentration in their vicinity, because it suppresses only the growth of normal cells and consequently, facilitates infiltration [16]. In short, the epithelium-only $\alpha v \beta 6$-integrin initiates and promotes the infiltrative growth of many kinds of malignant epithelial neoplasia-nowadays synonymous to 'carcinoma', a term that was originally coined about $400 \mathrm{BC}$ by Hippocrates ('karkinos' is greek for crayfish) and $\approx 400$ years later latinized by Celsus to 'cancer'. Of all integrins, expression of $\alpha v \beta 6$ is apparently most tightly connected to what is known ever since as the archetype of cancer, which is why we chose to refer to it as the 'cancer integrin' [17].

Consistent with its role of promoting invasion, the highest av $\beta 6$-integrin expression density is often found at the boundary of tumor- and healthy tissue [18]. There, it also drives tissue remodeling by epithelial-mesenchymal transition (EMT) via the TGF- $\beta$ pathway and the interaction with tumor stroma. Cells of stroma-rich neoplasia such as pancreatic ductal adenocarcinoma (PDAC) thus frequently show concomitant $\alpha v \beta 6$-integrin expression $[19,20]$. We found a high expression of the $\beta 6$ subunit on tumor cells in $88 \%$ of nearly 400 specimen of pancreatic ductal adenocarcinoma (PDAC) primaries, in 14 out of 15 metastases, and in $57 \%$ of grade 3 pancreatic intraepithelial neoplasia (PanIN3; immediate PDAC 
precursor lesions) [21]. Its prevalence in PDAC but also in other carcinomas, most importantly, squamous cell [18], basal cell, lung adeno, and colon [22] make it a highly attractive target for tumor imaging [23]. $\alpha v \beta 6$-integrin is furthermore expressed in the course of other conditions associated with TGF- $\beta$ driven extracellular matrix (ECM) remodeling such as idiopathic pulmonary fibrosis (IPF) [24], thereby further expanding the potential field of applications.
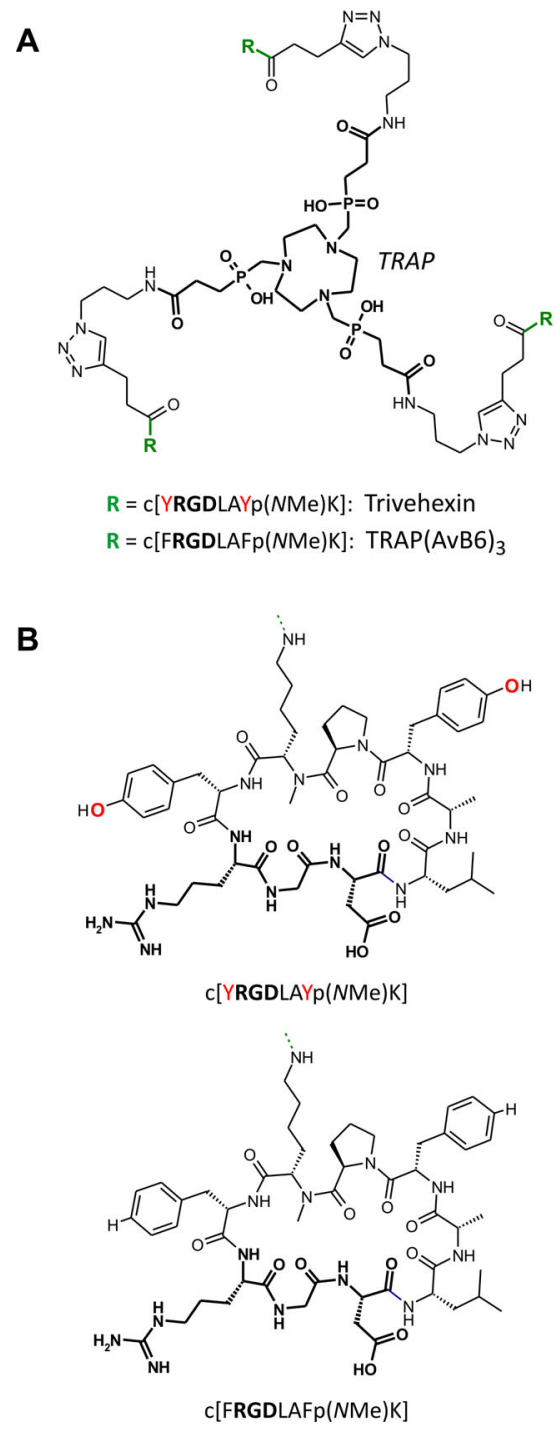

Figure 1: Chemical structures of peptides and conjugates. A, structure of the trimers $\operatorname{TRAP}(\operatorname{AvB6})_{3}$ and Trivehexin, which were synthesized by "click-chemistry" (i.e., copper(I) catalyzed azide-alkyne cycloaddition, CuAAC) conjugation of the TRAP chelator core (highlighted in boldface). B, Structures of the peptides used, with their RGD sequences highlighted in boldface. On an atomic level, the structural differences merely consists of two additional oxygens of c[YRGDLAYp(NMe)K] (colored in red).

A variety of $\alpha v \beta 6$-integrin targeted PET radiopharmaceuticals have therefore been developed in recent years. Some were already tested in humans [25,26,27,28,29] or even employed in clinical trials $[30,31,32]$, thus underscoring the clinical potential of $\alpha v \beta 6$-integrin imaging in human carcinomas and in IPF. Non-specific uptakes in stomach or intestines, and to a lesser extent, also in liver, lung or pancreas, might however challenge the respective diagnostic procedures. Such occasionally observed uptakes are prone to compromising the unambiguous identification of tumors and particularly of 
metastases, or raise the detection limit for low-level target expression. Along these lines, we earlier reported ${ }^{68} \mathrm{Ga}$-Avebehexin [17], a ${ }^{68} \mathrm{Ga}$-labeled monomeric triazacyclononane-triphosphinate (TRAP) [33] conjugate of $\alpha v \beta 6$-integrin-specific cyclic nonapeptide c[FRGDLAFp(NMe)K] [34], as well as a series of other ${ }^{68} \mathrm{Ga}$ - and ${ }^{177} \mathrm{Lu}$-labeled conjugates thereof [35]. Most of these compounds showed a very low background and thus, a good tumor-to-background contrast in rodent models, but their tumor accumulation was ultimately too low for a successful clinical transfer. Although we repeatedly observed that the trimeric ${ }^{68} \mathrm{Ga}$-TRAP conjugates of integrin ligands showed a much higher affinity, improved selectivity, enhanced tumor uptake, and prolonged retention [36,37,38,39,40], this strategy did not work out for c[FRGDLAFp(NMe)K]. A ${ }^{68} \mathrm{Ga}$-labeled TRAP trimer thereof, referred to as ${ }^{68} \mathrm{Ga}$-TRAP(AvB6) 3 (Figure 1), showed a very unfavorable biodistribution [17] and required further optimization. We now report the successful application of the novel, improved ${ }^{68}$ Ga-labeled trimerized $\alpha v \beta 6$-integrin selective nonapeptide ${ }^{68} \mathrm{Ga}$-Trivehexin (Figure 1) for imaging of human carcinomas, thus transforming the concept of multivalent molecular imaging probes from a future vision [41] into clinical reality.

\section{Materials and Methods}

\section{General}

Materials, reagents, general procedures, and instrumentation used for chemical syntheses and analyses have been detailed elsewhere [39]. In addition to what has been reported before, the building block TRAP(azide) 3 was purchased from Fosfinos s.r.o. (Kladno, CZ). c[FGDLAFp(NMe)K(pentynoic amide)] and TRAP(AvB6) $)_{3}$ were synthesized according to the literature [17]. Previously described protocols were followed for determination of integrin affinities by ELISA on immobilized integrins [42] and for $\beta 6$-integrin immunohistochemistry (IHC) [17].

\section{Compounds}

\section{c[YRGDLAYp(NMe)K(pentynoic amide)]}

General. The linear peptide sequence was assembled on 2-chlorotrityl resin (CC11006 by Carbolution, Germany), applying standard Fmoc strategy in $20 \mathrm{~mL}$ filter syringes with anhydrous dimethyl formamide (DMF) as solvent (except for resin loading, where dichloromethane (DCM) was used). For coupling, Fmoc-amino acids (2 eq) were dissolved in DMF ( $5 \mathrm{~mL}$ ), pre-activated for 10 min with HATU ( 2 eq), HOBt ( 2 eq), and diisopropylethylamine (DIPEA, 6 eq), and gently shaken with the resin for a minimum of $3 \mathrm{~h}$. Completeness of each coupling step was monitored by LC-MS after cleaving off a small sample of resin with a solution of 1,1,1,3,3,3-hexafluoropropane-2-ol (HFIP, 20\% by volume) in DMF. Fmoc deprotection was done by treating the resin $2 \times$ for 10 min with DMF containing $20 \%(\mathrm{v} / \mathrm{v})$ piperidine $(6 \mathrm{~mL})$; the resin was washed $4 \times$ with DMF before and after this step.

Resin loading and peptide assembly. $1 \mathrm{~g}$ resin was loaded with Fmoc-Gly (approx. loading capacity: $0.9 \mathrm{mmol})$, capped with a mixture of methanol $(\mathrm{MeOH})$ and DIPEA (2:1 by volumes), and washed with 
DCM and DMF (4× each). Then, Fmoc-L-Arg(Pbf)-OH, Fmoc-L-Tyr $(t \mathrm{Bu})-\mathrm{OH}$, and Fmoc-L-Lys(Dde)$\mathrm{OH}$ were coupled with intermediate Fmoc deprotection.

$\mathrm{N}$-methylation. After Fmoc removal from Lys, $\mathrm{N}$-methylation was done via the Mitsunobu reaction. First, an $o$-NBS protection group was installed at the $N$-terminus by treating the resin with a solution of 2-nitrobenzenesulfonylchloride (o-NBS-Cl, 4 eq) and 2,4,6-collidine (10 eq) in DCM for 20 min. After washing with DCM $(3 \times)$ and anhydrous tetrahydrofurane (THF; $5 \times)$, the resin was immersed in a solution of triphenylphosphine $\left(\mathrm{PPh}_{3}, 5 \mathrm{eq}\right)$ in anhydrous $\mathrm{MeOH}$, to which a solution of diisopropyl azodicarboxylate (DIAD, 5 eq) in a minimal amount of dry tetrahydrofurane (THF) was slowly added. After $15 \mathrm{~min}$, the resin was washed with THF $(5 \times)$ and DMF $(5 \times)$, and the $o$-NBS protection group removed with 2-mercaptoethanol (10 eq) and 1,8-diazabicyclo[5.4.0]undec-7-ene (DBU) in DMF $(6 \mathrm{~mL})$ for $5 \mathrm{~min}$.

Peptide assembly and cleavage. After washing with DMF $(5 \times)$, coupling was continued with Fmoc-DPro-OH, Fmoc-L-Tyr $(t \mathrm{Bu})-\mathrm{OH}$, Fmoc-L-Ala-OH, Fmoc-L-Leu-OH, and Fmoc-L-Asp $(t \mathrm{Bu})-\mathrm{OH}$. The protected linear peptide was cleaved from the resin with a solution of HFIP ( $20 \%$ by volume) in DCM $(7 \mathrm{~mL})$. Cleavage was repeated $2 \times$, the resin washed with DCM $(5 \times)$, all solutions combined and concentrated in vacuo.

Cyclization. To the crude linear peptide, DMF was added to reach a concentration of $1 \mathrm{mM}$, and vigorously stirred with anhydrous $\mathrm{NaHCO}_{3}(5 \mathrm{eq})$ and diphenylphosphorylazide (DPPA, 3 eq) for $2 \mathrm{~d}$ at ambient temperature. The solution was concentrated in vacuo to $100 \mathrm{~mL}$, filtered, and the Dde protecting group removed by addition of hydrazine hydrate $(2 \mathrm{~mL})$. Thereafter, the peptide intermediate was purified on a Biotage flash chromatography station on C18-RP cartridges, typically affording approx. $250 \mathrm{mg}(\approx 20 \%$ of theoretical yield).

Alkyne functionalization. A solution of 4-pentynoic acid (1.5 eq), HATU (1.5 eq), HOBt (1.5 eq), and DIPEA ( 2 eq) in a minimal amount of DMF was left to react for $15 \mathrm{~min}$ and added dropwise to a solution of the peptide intermediate (1 eq) and DIPEA (1 eq) in DMF. After $1 \mathrm{~h}$ at ambient temperature, the solvent was evaporated and the residue treated with a mixture of trifluoroacetic acid / DCM / triisopropylsilane / $\mathrm{H}_{2} \mathrm{O}$ (85:10:2.5:2.5 by volumes) for up to $3 \mathrm{~h}$ until LC-MS monitoring indicated complete deprotection. The deprotected peptide was precipitated into diethyl ether and purified by preparative HPLC, typically yielding $>100 \mathrm{mg}$ of the title compound. MW (calcd. for $\mathrm{C}_{56} \mathrm{H}_{79} \mathrm{~N}_{13} \mathrm{O}_{14}$ ): 1158.33. ESI-MS (positive mode): $m / z=1932.1\left[5 \mathrm{M}+3 \mathrm{H}^{+}\right]^{3+}, 1739.0\left[3 \mathrm{M}+2 \mathrm{H}^{+}\right]^{2+}, 1159.1\left[\mathrm{M}+\mathrm{H}^{+}\right]^{+}$, $580.4\left[\mathrm{M}+2 \mathrm{H}^{+}\right]^{2+}$ (for MS spectra see Supporting Information, Figure S2).

\section{Trivehexin}

c[YRGDLAYp(NMe)K(pentynoic acid)] $(24.9 \mathrm{mg}, 21.5 \mu \mathrm{mol}, 3.3 \mathrm{eq})$ was added to a solution of TRAP(azide) $)_{3}(5.4 \mathrm{mg}, 6.5 \mu \mathrm{mol}, 1 \mathrm{eq})$ and sodium ascorbate ( $\left.65 \mathrm{mg}, 326 \mu \mathrm{mol}, 50 \mathrm{eq}\right)$ in a minimal amount of $\mathrm{H}_{2} \mathrm{O} . \mathrm{Cu}^{\text {II }}$ acetate $(1.56 \mathrm{mg}, 7.84 \mu \mathrm{mol}, 1.2 \mathrm{eq})$ was added and a brown precipitate immediately formed. Upon vortexing, the solution turned to a transparent green. The solution reacted for $1 \mathrm{~h}$ at $60{ }^{\circ} \mathrm{C}$ without stirring. After $1 \mathrm{~h}, \mathrm{Cu}^{\mathrm{II}}$ was removed from the TRAP chelator by reaction with 
a solution of 1,4,7-triazacyclononane-1,4,7-triacetic acid (NOTA) (39 mg, $130 \mu \mathrm{mol}, 20$ eq.) in water $(1 \mathrm{~mL})$, adjusted to $\mathrm{pH} 2.2$ by addition of $1 \mathrm{M}$ aq HCl, for $1 \mathrm{~h}$ at $60{ }^{\circ} \mathrm{C}$. Purification by preparative RPHPLC (gradient: $20-40 \% \mathrm{MeCN}$ in $\mathrm{H}_{2} \mathrm{O}$, both containing $0.1 \%$ TFA, in $15 \mathrm{~min}$ ) yielded $10.1 \mathrm{mg}$ Trivehexin (36\%). MW (calcd. for $\mathrm{C}_{195} \mathrm{H}_{291} \mathrm{~N}_{54} \mathrm{O}_{51} \mathrm{P}_{3}$ ): 4300.75. ESI-MS (positive mode): $\mathrm{m} / z=1721.5$ $\left[2 \mathrm{M}+5 \mathrm{H}^{+}\right]^{5+}, 1434.7\left[\mathrm{M}+3 \mathrm{H}^{+}\right]^{3+}, 1076.2\left[\mathrm{M}+4 \mathrm{H}^{+}\right]^{4+}, 861.1\left[\mathrm{M}+5 \mathrm{H}^{+}\right]^{5+}, 717.8\left[\mathrm{M}+6 \mathrm{H}^{+}\right]^{6+}$. The ${ }^{\text {nat }} \mathrm{Ga}-$ complex of Trivehexin for determination of affinities formed immediately upon mixing equal amounts $(100 \mu \mathrm{L})$ of $1 \mathrm{mM}$ aq. solutions of Trivehexin and $\mathrm{Ga}\left(\mathrm{NO}_{3}\right)_{3}$ hydrate. MW (calcd. for $\mathrm{C}_{195} \mathrm{H}_{288} \mathrm{~N}_{54} \mathrm{O}_{51} \mathrm{P} 3 \mathrm{Ga}$ ): 4367.44. ESI-MS (positive mode): $m / z=1747.9\left[2 \mathrm{M}+5 \mathrm{H}^{+}\right]^{5+}, 1456.8\left[\mathrm{M}+3 \mathrm{H}^{+}\right]^{3+}$, $1092.7\left[\mathrm{M}+4 \mathrm{H}^{+}\right]^{4+}, 874.4\left[\mathrm{M}+5 \mathrm{H}^{+}\right]^{5+}, 728.9\left[\mathrm{M}+6 \mathrm{H}^{+}\right]^{6+}$ (for MS spectra see Supporting Information, Figure S4).

\section{Radiolabeling}

Radiolabeling for preclinical experiments and determination of $n$-octanol-PBS distribution coefficients ( $\left.\log D_{7.4}\right)$ was done as described [39]. Applying this protocol, $5 \mathrm{nmol}(0.5 \mathrm{nmol}$ for cellular assays in order to maximize molar activity) of $\operatorname{TRAP}(\mathrm{AvB} 6)_{3}$ or Trivehexin were labeled with ${ }^{68} \mathrm{Ga}$ at $\mathrm{pH} 2$, resulting in RCY $>95 \%$. The purity of the radiolabeled compounds was confirmed by radio-TLC, using silica impregnated glass fiber chromatography paper (ITLC ${ }^{\circledR}$ by Agilent) as stationary phase, and $0.1 \mathrm{M}$ aq. sodium citrate as mobile phase (purities were $>99 \%$ ).

Radiolabeling for clinical testing was performed by an automated procedure on a Modular-Lab module (Eckert \& Ziegler, Berlin) connected to a ${ }^{68} \mathrm{Ge} /{ }^{68} \mathrm{Ga}-$ Generator (GalliaPharm ${ }^{\circledR}$ by Eckert \& Ziegler, Berlin, Germany) in a sterile hot cell as described earlier [40]. The final product (450 $\pm 45 \mathrm{MBq}$, starting from $\sim 600 \mathrm{MBq}$ ) was diluted with $0.15 \mathrm{M}$ phosphate-buffered saline $(9 \mathrm{~mL}$; SC-01-PbS, $\mathrm{ABX}$, Radeberg, Germany). The QC release citeria (endotoxin level $<5.00 \mathrm{EU} / \mathrm{mL}$; radio-TLC $>99 \%$; radioHPLC > 95\%; pH 4.0-8.0) were met for all radiosyntheses. Sterility was tested retrospectively after complete decay ( $3 \mathrm{~d}$ post synthesis).

\section{Cellular uptake assay}

H2009 human lung adenocarcinoma cells (CRL-5911) and MDA-MB-213 cells (HTB-26; both from American Type Culture Collection (ATCC), Manassas, VA, USA) were cultivated as recommended by the distributor. Cells were grown in a monolayer culture at $37{ }^{\circ} \mathrm{C}$ in a $5 \% \mathrm{CO}_{2}$ humidified atmosphere. All cells were tested regularly to exclude mycoplasma contamination.

$\alpha v \beta 6$-dependent uptake of ${ }^{68} \mathrm{Ga}$-Trivehexin was determined in H2009 ( $\alpha \mathrm{v} \beta 6$-positive) and MDA-MB231 ( $\alpha$ v $\beta 6$-negative) cells. 100,000 cells per well were seeded into a 96-well plate and incubated with a $0.3 \mathrm{nM}$ solution of ${ }^{68} \mathrm{Ga}$-Trivehexin (30 kBq/well; molar activity: $\left.452-558 \mathrm{MBq} / \mathrm{nmol}\right)$ for $1 \mathrm{~h}$ at $37{ }^{\circ} \mathrm{C}$. For blocking, ${ }^{\text {nat }} \mathrm{Ga}$-Trivehexin was added prior to the radioligand at a final concentration of $250 \mathrm{nM}$. At the end of the incubation time, cells were pelleted and washed twice, followed by cell lysis with a $1 \mathrm{M}$ $\mathrm{NaOH}$ solution. Supernatants and cell lysates were collected and measured on a Wizard ${ }^{2}$ automated 
gamma counter (PerkinElmer). The cellular uptake of ${ }^{68} \mathrm{Ga}$-Trivehexin was quantified as percent of total radioactivity added. This experiment was repeated $6 \times(\mathrm{H} 2009)$ or $4 \times($ MDA-MB-213) with 5 or 3 technical replicates, respectively.

\section{Animal experiments}

All animal experiments were approved by the responsible authority (Regierung vonn Oberbayern) and have been performed in accordance with general animal welfare regulations in Germany and the institutional guidelines for the care and use of animals. Keeping of the animals, generation of subcutaneous H2009 and MDA-MB-231 tumor xenografts, ex-vivo biodistribution studies, and $\mu$ PET imaging were done according to previously described protocols [39]. Multiplicity of PET and biodistribution studies ranged from 3 to 5 ; animal numbers for each individual experiment are denoted in the respective data tables in the Supporting Information and, additionally, in the Results section.

\section{Patients}

The synthesis and quality control of ${ }^{68} \mathrm{Ga}$-Trivehexin for clinical application was done applying a previously described protocol [40], using $10 \mathrm{nmol}$ of Trivehexin. Application of ${ }^{68} \mathrm{Ga}$-Trivehexin in human was done according to $\S 13 / 2 b$ of the German Drug Act (Arzneimittelgesetz). All human participants provided written informed consent, also for publication of images, prior to the investigation. Following i.v. administration of ${ }^{68} \mathrm{Ga}$-Trivehexin, there were no adverse or clinically detectable pharmacologic effects. No significant changes in vital signs or the results of laboratory studies or electrocardiograms were observed. Data for a total of 4 human subjects were included in this report. The PET/CT for patient \#1 was performed according to a previously published protocol, as was calculation of the dosimetric parameters [40].

Patient \#2 (Figure 4A+B) underwent both $\left[{ }^{18} \mathrm{~F}\right] \mathrm{FDG}$ and ${ }^{68} \mathrm{Ga}$-Trivehexin PET/CT within 2 weeks for primary staging of a recently detected, locally advanced p-16 negative head-and-neck squamous cell carcinoma (HNSCC) of the oropharynx with infiltration of the lips and ipsilateral cervical lymph node metastases (cT4a cN2b cM0). A pandendoscopy and tracheostomy was carried out four weeks prior to the $\left[{ }^{18} \mathrm{~F}\right] \mathrm{FDG}$. The tumor was not suitable for surgical removal and a simultaneous radiochemotherapy was indicated.

Patient \#3 (Figure 4C-E) was diagnosed with an adenocarcinoma (salivary duct carcinoma) in the right parotis in 2019 and initially underwent total parotidectomy and unilateral neck dissection (pT2 pN1b [21/23] L1 V1 Pn1 R1). A post-resection and contralateral neck-dissection was performed one month later, followed by adjuvant radiation. In 2020 the patient developed prelaryngeal and lateral orbita angle metastases, which were resected and adjuvantly irradiated. A follow-up MRI revealed a new subcutaneous prelaryngeal metastasis. Consecutively the patient underwent $\left[{ }^{18} \mathrm{~F}\right] \mathrm{FDG}$ and ${ }^{68} \mathrm{Ga}-$ Trivehexin PET/CT within two days. Since no further tumor manifestations were present in both 
examinations, surgical resection was indicated and performed. $\beta 6$-integrin IHC [21] was done for specimen of the excised metastasis.

Patient \#4 was diagnosed with a carcinoma of the pancreatic head by endosonography and CT morphology. Pancreatic adenocarcinoma was verified by biopsy. A PET/CT scan using ${ }^{68} \mathrm{Ga}$-Trivehexin was performed 120 min after i.v. administration, showing a high tracer uptake in the pancreatic tumor burden and multiple liver metastases. According to this finding, surgical treatment was rejected and a chemotherapy regimen was started.

\section{Results}

\section{Synthesis and in-vitro characterization}

Similar to TRAP(AvB6) $)_{3}[17]$ or the previously reported $\alpha v \beta 8$-integrin tracer ${ }^{68}$ Ga-Triveoctin [40], the trimeric conjugate Trivehexin was synthesized by conjugation of the Lys(pentynoic amide) derivative of $\mathrm{c}[\mathrm{YRGDLAYp}(N \mathrm{Me}) \mathrm{K}]$ to the symmetrical TRAP chelator scaffold [43] (TRAP $=1,4,7-$ triazacyclononane-1,4,7-tris[methylene(2-carboxyethyl)]phosphinic acid) [44], applying a convenient and straightforward click chemistry (CuAAC) protocol [45] (Figure 1). Automated ${ }^{68} \mathrm{Ga}$ labeling of $5 \mathrm{nmol}$ of Trivehexin for preclinical characterization afforded ${ }^{68} \mathrm{Ga}$-Trivehexin with radiochemical yields exceeding $95 \%$ and purity (radio-TLC) of $>99 \%$.

Table 1: Integrin affinities, expressed as $50 \%$ inhibition constants $\left(I C_{50}\right)$, and $I C_{50}$-based selectivities.

\begin{tabular}{|c|c|c|c|c|c|c|c|}
\hline \multirow[t]{2}{*}{ Compound } & \multicolumn{4}{|c|}{$I C_{50}(95 \%$ confidence interval $)[\mathrm{nM}]$} & \multicolumn{3}{|c|}{$\alpha v \beta 6$-selectivity over } \\
\hline & $\alpha v \beta 6$ & $\alpha v \beta 8$ & $\alpha v \beta 3$ & $\alpha 5 \beta 1$ & $\alpha v \beta 8$ & $\alpha v \beta 3$ & $\alpha 5 \beta 1$ \\
\hline $\mathrm{c}[\mathrm{FRGDLAFp}(N \mathrm{Me}) \mathrm{K}$ (pentynoic amide)] & $0.36(0.20-0.69)$ & $32(20-51)$ & $424(270-670)$ & $226(115-193)$ & 89 & 153 & 81 \\
\hline$c[$ YRGDLAYp $(N \mathrm{Me}) \mathrm{K}$ (pentynoic amide)] & $1.7(1.2-2.6)$ & $26(19-37)$ & $219(88-540)$ & $150(116-193)$ & 15.3 & 128 & 88 \\
\hline${ }^{\text {nat }}$ Ga-Trivehexin & $0.033(0.008-0.13)$ & $6.2(2.8-14)$ & $2.7(1.2-6.0)$ & $22(16-30)$ & 188 & 82 & 667 \\
\hline
\end{tabular}

The exchange of both phenylalanines in $\mathrm{c}[\mathrm{FRGDLAFp}(N \mathrm{Me}) \mathrm{K}]$ to tyrosines was done with the intention to increase the hydrophilicity of the resulting conjugates, because we observed earlier that TRAP$\mathrm{c}[\mathrm{FRGDLAFp}(\mathrm{NMe}) \mathrm{K}]$ trimers possessed two-digit picomolar $\alpha \mathrm{v} \beta 6$-integrin affinities but were essentially unusable because of the concomitant increase of non-specific organ uptakes [17]. Replacement of each individual Phe in c[FRGDLAFp $(N \mathrm{Me}) \mathrm{K}]\left(I C_{50}=0.26 \mathrm{pM}\right)$ [34) by Tyr was shown earlier to result in only slightly lower affinities $\left(I C_{50}\right.$ of 0.39 and $0.40 \mathrm{pM}$ for c[FRGDLAYp $(N \mathrm{Me}) \mathrm{K}$ ] and c[YRGDLAFp(NMe)K]), respectively) [34]. Simultaneous Phe-to-Tyr exchange at both positions lead to a notably lower $I C_{50}$ and reduced subtype selectivities of the building block c[YRGDLAYp(NMe)K(pentynoic amide)], as compared to its phenylalanine congener (see Table 1). This drawback was however compensated by trimerization, which, in accordance with previous observations [39], resulted in a substantially higher $\alpha v \beta 6$-integrin affinity (52-fold) and a substantial 
attenuation of some selectivity factors (Table 1). Finally, ${ }^{68} \mathrm{Ga}$-Trivehexin also showed the desired increase in hydrophilicity, its $n$-octanol-PBS distribution coefficient $\left(\log D_{7.4}=-2.1 \pm 0.1\right)$ being moderately lower than that of ${ }^{68} \mathrm{Ga}-\mathrm{TRAP}(\mathrm{AvB} 6)_{3}$ (re-determined, $\log D_{7.4}=-1.5 \pm 0.1$ ).

\section{Preclinical evaluation}

A

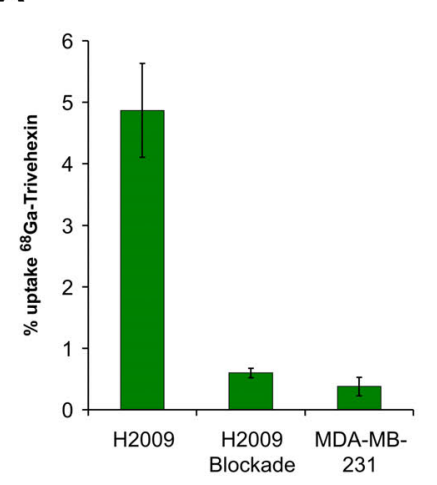

B

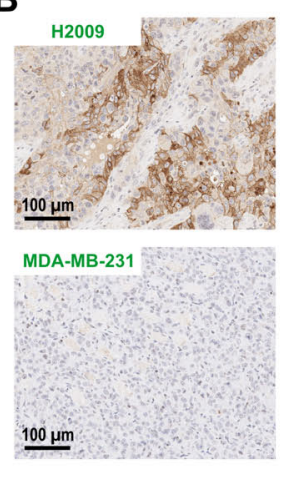

C

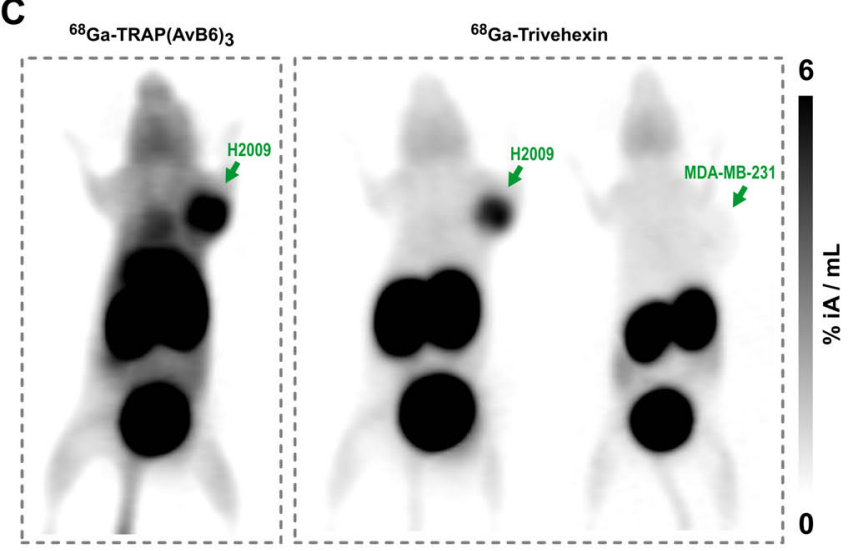

D

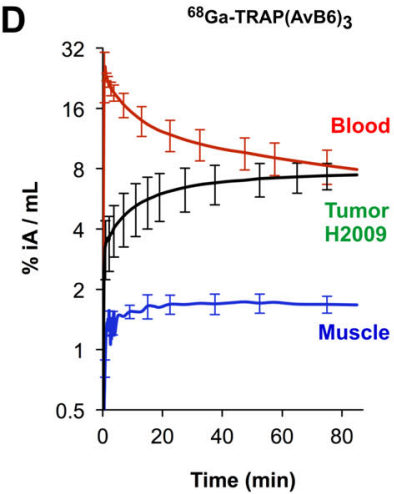

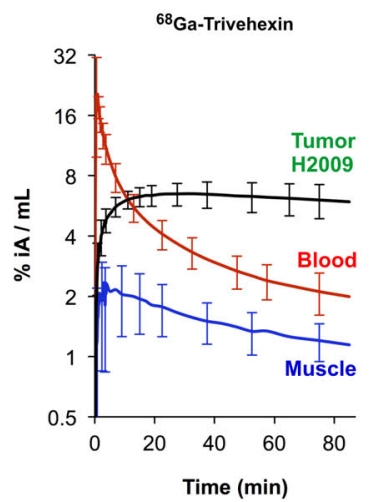

E

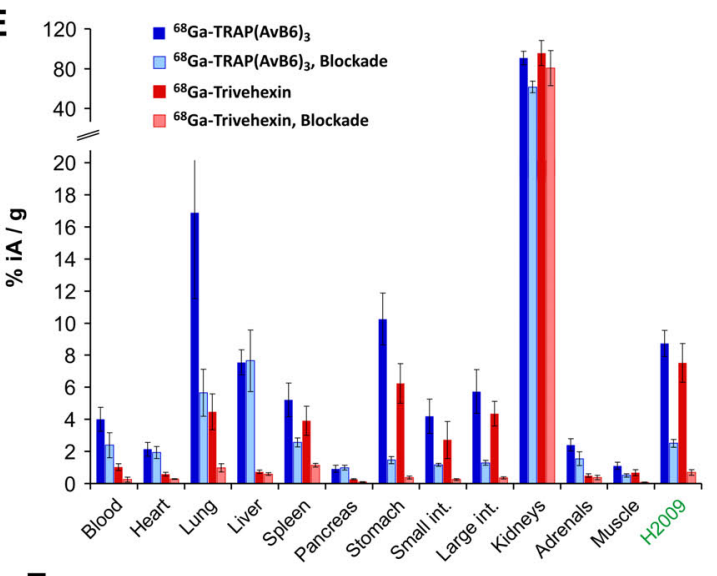

$\mathbf{F}$

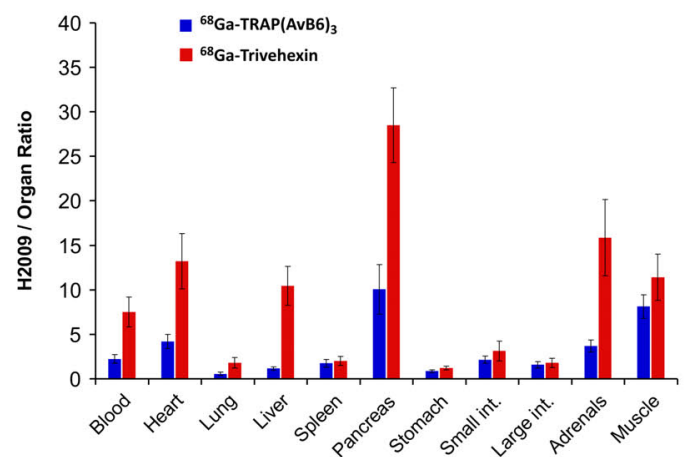

G

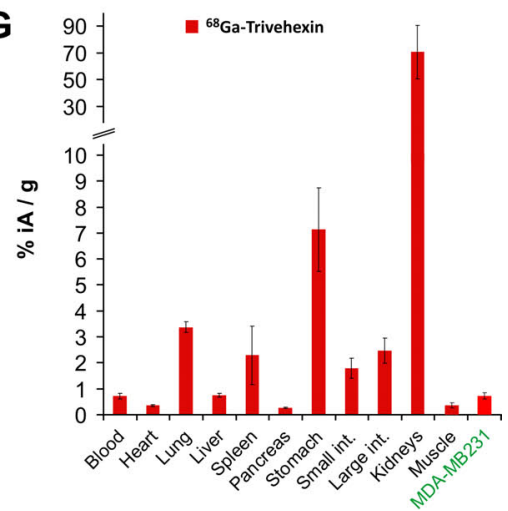

Figure 2. Preclinical development of ${ }^{68} \mathrm{Ga}$-Trivehexin. Data were generated using $\mathrm{H} 2009$ ( $\alpha \vee \beta 6^{+}$human lung adenocarcinoma) or MDA-MB-231 ( $\alpha v \beta 6^{-}$human triple-negative breast cancer) cell lines, and murine xenografts thereof. A, Cellular uptake of ${ }^{68} \mathrm{Ga}$-Trivehexin with and without blocking with ${ }^{\text {nat }} \mathrm{Ga}$-Trivehexin. Mean and SD of $\mathrm{n}=4-6$ biological repeats. B, $\beta 6$-integrin immunohistochemistry (IHC) confirms moderate $\alpha v \beta 6$-integrin expression in $\mathrm{H} 2009$ and absence of $\alpha \vee \beta 6$ in MDA-MB-231 tumor xenografts. C, Static $\mu$ PET images (maximum intensity projections), 75 min p.i., recording time $20 \mathrm{~min}$. The same $\mathrm{H} 2009$-tumor bearing animal was used for both PET scans shown, with a $24 \mathrm{~h}$ recovery period. D, Biokinetics of ${ }^{68} \mathrm{Ga}$-labeled trimers, derived from dynamic PET scans. E, Biodistribution of trimers in H2009-bearing animals. Dark bars indicate controls (injected molar 
amounts of approx. $100 \mathrm{pmol}, n=5$ ); light bars of the same color show blockade (50 nmol unlabeled, administered $10 \mathrm{~min}$ before the radioactive compound, $n=3$ ). F, Tumor-to-organ ratios derived from H2009 biodistribution data. G, Biodistribution of ${ }^{68} \mathrm{Ga}$-Trivehexin (105 \pm 34 pmol) in MDA-MB-231-bearing animals $(n=$ 5). - Numerical data for graphs shown in E, F, and G, including the exact values of injected amounts, are provided in the Supporting Information, Tables S1-3.

The $\alpha v \beta 6$-integrin expressing human lung adenocarcinoma cell line $\mathrm{H} 2009$ and the $\alpha v \beta 6$-negative breast cancer cell line MDA-MB-231 were used for cellular assays and in-vitro experiments. ${ }^{68} \mathrm{Ga}$-Trivehexin showed specific (i.e. blockable) uptake in $\mathrm{H} 2009$ but not in MDA-MB-231 cells (Figure 2A). Both ${ }^{68} \mathrm{Ga}-$ labeled trimers were further evaluated in severe combined immunodeficient (SCID) mice, bearing subcutaneous xenografts of the same cell lines on the right shoulders. Dynamic $\mu$ PET revealed that ${ }^{68} \mathrm{Ga}-$ Trivehexin was cleared much faster from the blood than ${ }^{68} \mathrm{Ga}-\mathrm{TRAP}(\mathrm{AvB} 6)_{3}$ (Figure 2D). In addition, it showed virtually no unfavorable non-specific accumulation (i.e., non-blockable uptake) in many organs, which was observed for ${ }^{68} \mathrm{Ga}-\mathrm{TRAP}(\mathrm{AvB} 6)_{3}$ particularly in the liver and the lung, but also in the tumor tissue (Figure 2E).

Of note, there were still blockable ${ }^{68} \mathrm{Ga}$-Trivehexin uptakes in e.g. lung and stomach, which are related to proven physiological $\beta 6$-integrin expression in mice [17]. The improved biodistribution profile translated to substantially enhanced tumor-to-organ ratios for ${ }^{68} \mathrm{Ga}$-Trivehexin (Figure $2 \mathrm{~F}$ ), and furthermore to $\mu \mathrm{PET}$ images with lower background and improved tumor delineation (Figure $2 \mathrm{C}$ ). Specificity for $\alpha v \beta 6$-expressing tumors was confirmed by PET and biodistribution in MDA-MB-231 xenografts. $\beta 6$-IHC confirmed that in contrast to H2009 tumors, MDA-MB-231 xenografts were $\beta 6$ negative (Figure 2B), which is why virtually no ${ }^{68} \mathrm{Ga}$-Trivehexin uptake was observed in PET and biodistribution (Figure $2 \mathrm{C}+\mathrm{G}$ ). 


\section{In-human dosimetry estimate}
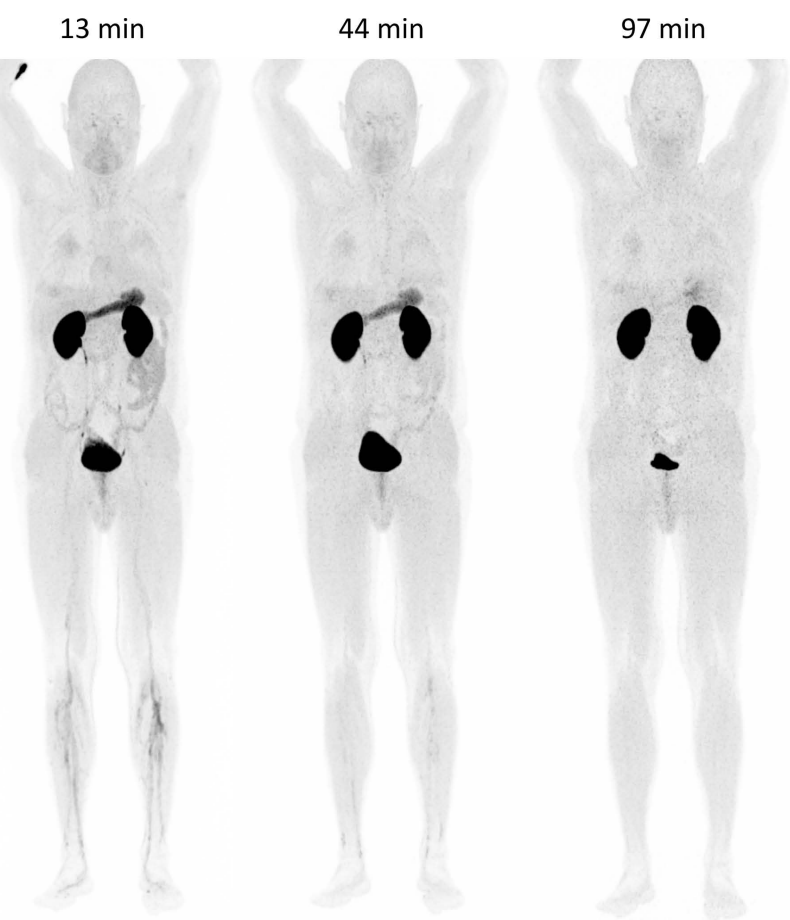

Figure 3: ${ }^{68} \mathrm{Ga}$-Trivehexin PET of patient \#1, $172 \mathrm{MBq}$, total peptide amount: $5 \mathrm{nmol}$, start times p.i. denoted above anterior maximum intensity projections (MIPS) scaled to SUV 15. Apart from kidney and urinary bladder, a significant uptake is noted in the (empty) stomach, which however had disappeared $1.5 \mathrm{~h}$ after injection. PET at $1 \mathrm{~h}$ p.i. or later is thus preferred. For organ SUVs and dosimetry data, see Supporting Information, Tables S4 and S5.

Whole-body PET images (Figure 3) showed a biodistribution generally characterized by a low soft tissue uptake. Apart from the renal excretion and partial retention, relevant uptakes were registered only in the gastric mucosa and the small intestine (for SUV values see Supporting Information Table S4). Currently, we are not able to provide a rationale for the transient uptake in the upper gastrointestinal tract, which decreased analogously to the vascular tracer retention rapidly over time and was nearly absent in the late image. We thus found that ${ }^{68} \mathrm{Ga}$-Trivehexin PET should ideally be performed at time points $>60$ min p.i.. With a biological half life of $10 \mathrm{~h}$ (renal excretion) and an assumed urinary bladder residence of $0.07 \mathrm{~h}$, dosimetry calculations using OLINDA V1.1 yielded a moderate effective dose (ICRP 60) of 3.36E-02 mSv/MBq, or $4.7 \mathrm{mSv}$ for an injection of $148 \mathrm{MBq}(4 \mathrm{mCi}){ }^{68} \mathrm{Ga}-T$ rivehexin (for individual organ dosimetry factors see Supporting Information Table S5).

\section{Imaging of carcinomas in human}

The tumor configuration and tracer uptake of both a locally advanced oropharyngeal HNSCC (Figure $3 \mathrm{~A}+\mathrm{B})$ and a prelaryngeal cutaneous metastasis of an initially resected right sided parotideal adenocarcinoma (Figure 3C+D) appear quite similar in the ${ }^{68}$ Ga-Trivehexin- and $\left[{ }^{18} \mathrm{~F}\right]$ FDG-PET. In the HNSCC patient \#2, the inflammatory, peristomal uptake around the tracheostoma was lower in the ${ }^{68} \mathrm{Ga}-$ Trivehexin study as compared to the $\left[{ }^{18}\right.$ F]FDG-PET done 2 weeks before. Reactively increased 
lymphatic uptake in the pharyngeal tonsil as well as in periclavicular, mediastinal and hilar lymph nodes occurred only in $\left[{ }^{18} \mathrm{~F}\right] \mathrm{FDG}$ imaging.

The prelaryngeal cutaneous metastasis of the adenocarcinoma patient \#3 (Figure 4D) showed a higher uptake of ${ }^{68} \mathrm{Ga}$-Trivehexin $\left(\mathrm{SUV}_{\max }=7.6\right)$ than of $\left[{ }^{18} \mathrm{~F}\right] \mathrm{FDG}\left(\mathrm{SUV}_{\max }=6.1\right)$, while both PET modalities indicated a metabolic tumor volume of $2.2 \mathrm{~cm}^{3}$. Immunohistochemistry for $\beta 6$-Integrin (Figure 4E) revealed a moderate cytoplasmic to membraneous positivity in $10 \%$ of the tumor cells with an increased ratio of positive neoplastic cells $(25 \%)$ towards the infiltrative margins of the metastasis. This is consistent with the observation that head-and-neck carcinomas express $\alpha v \beta 6$-integrin particularly at the invasive edge where tumor cells are in direct contact with the tumor-associated stroma [18]. In addition, both tracers showed a comparably low bifocal uptake in the mastoid cells on the right side, representing an inflammatory process, and a modest physiological uptake in the breast parenchyma.

The PET/CT shown in Figure 5 is presenting two parts of a pancreatic tumor of patient \#4, one placed in the region of pancreatic head passing over to the corpus, and the other part more lateral passing from the corpus to the tail. Presence of tumor lesions was described by a CT-only scan before, whereby one metastatic lesion was discovered in liver segment 8 . Other liver lesions were interpreted as typical cysts by $\mathrm{CT}$ only, but could be identified as PDAC metastases by ${ }^{68} \mathrm{Ga}$-Trivehexin PET positivity. Of note, this scan was performed with a comparably low activity of $105 \mathrm{MBq}$ and at a late time point of $120 \mathrm{~min}$, i.e., after nearly two ${ }^{68} \mathrm{Ga}$ half lives. Figure 5 thus demonstrates the capabilities of ${ }^{68} \mathrm{Ga}$-Trivehexin PET, because clinically relevant information could be obtained from the images despite the low remaining total activity at scan time (approx. $30 \mathrm{MBq}$, less urinary excretion) which caused more background noise than in the PET images shown in Figures 4 and 5. 
A

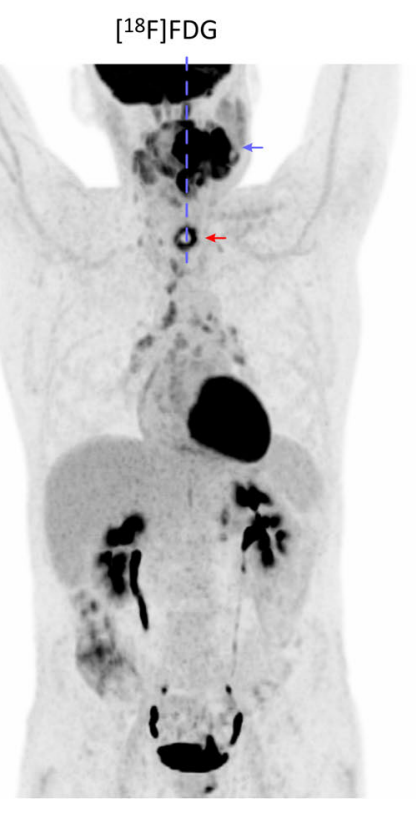

C

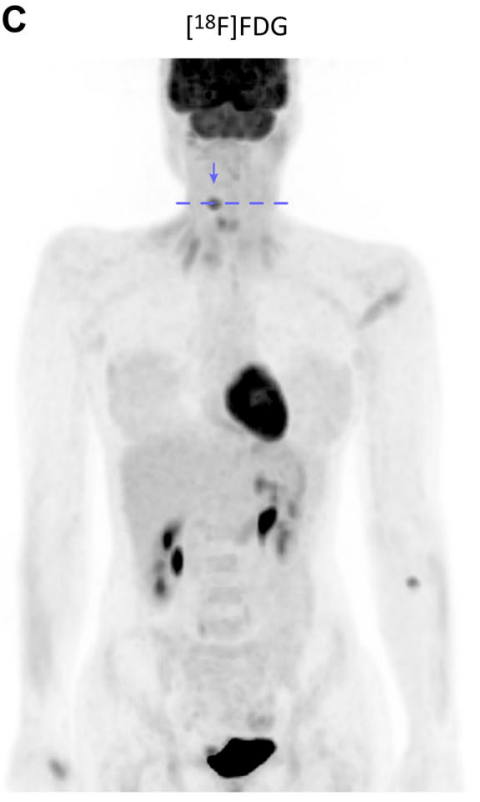

${ }^{68} \mathrm{Ga}$-Trivehexin

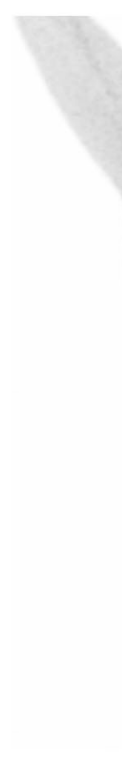

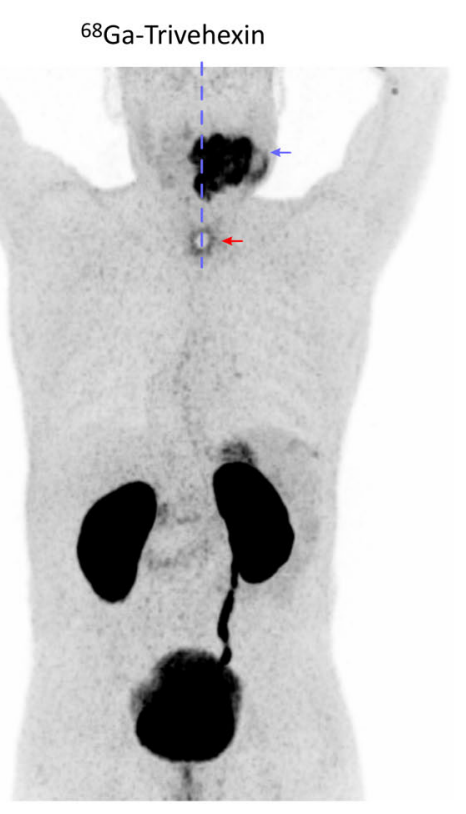

B

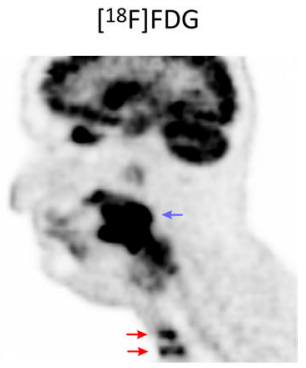

${ }^{68} \mathrm{Ga}$-Trivehexin

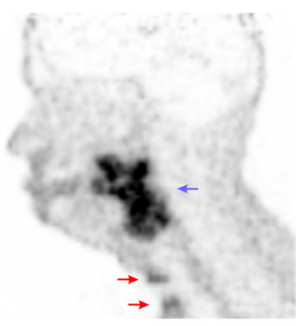

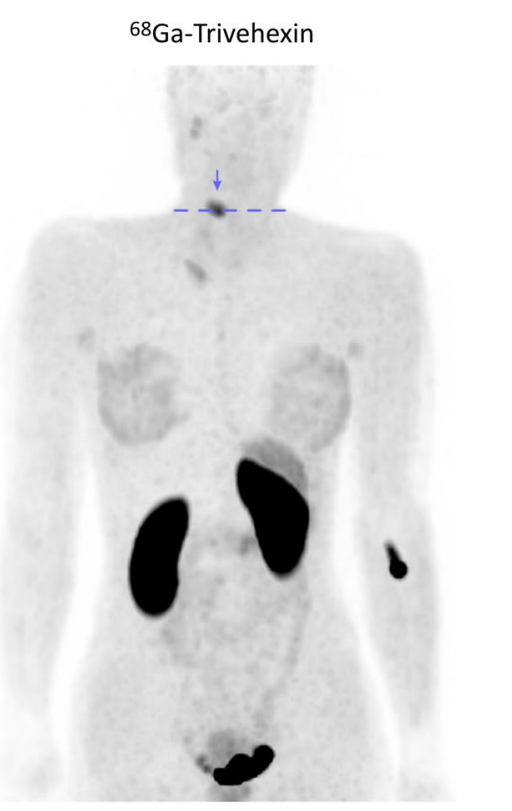

D

$[18 \mathrm{~F}] \mathrm{FDG}$

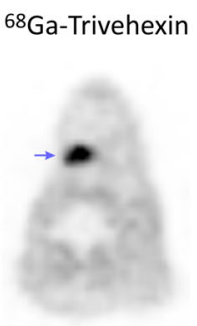

E
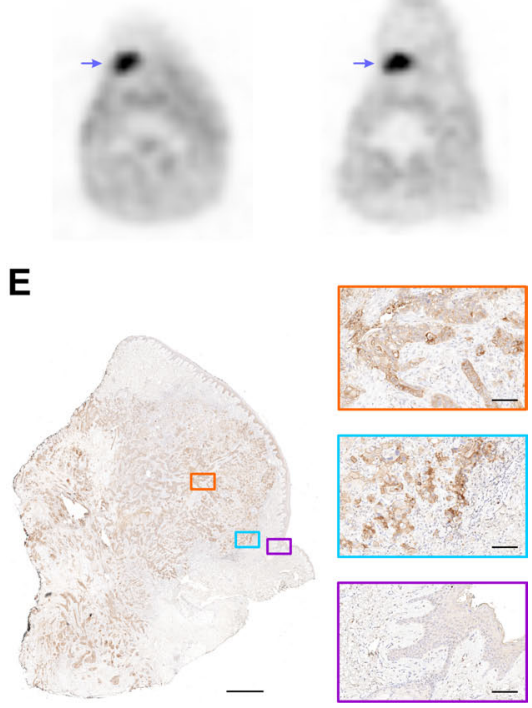

Figure 4: $\left[{ }^{18} \mathrm{~F}\right] \mathrm{FDG}$ and corresponding ${ }^{68} \mathrm{Ga}$-Trivehexin PET of head-and-neck cancers (locations indicated by blue arrows, slice planes by blue dashed lines). All PET images are scaled to SUV 8, except D (to SUV 5). A, PET (MIPS) of HNSCC patient \#2 (m, 61 y, $70 \mathrm{~kg} ; 331 \mathrm{MBq}\left[{ }^{18} \mathrm{~F}\right] \mathrm{FDG} 62 \min$ p.i.; $142 \mathrm{MBq}{ }^{68} \mathrm{Ga}$-Trivehexin, $A_{\mathrm{M}}=27 \mathrm{MBq} / \mathrm{nmol}$, total peptide amount $5.3 \mathrm{nmol}, 61 \mathrm{~min}$ p.i.) with locally advanced oropharyngeal carcinoma and several adjacent unilateral lymph node metastases. ${ }^{68} \mathrm{Ga}$-Trivehexin showed uptake in the primary and metastases $\left(\mathrm{SUV}_{\max }=13.9\right.$ and 12.9, respectively), and in a lesser extent around a 7-week old tracheostoma (red arrows, $\operatorname{SUV}_{\max }=5.8$; cf. 10.2 for $\left[{ }^{18} \mathrm{~F}\right] \mathrm{FDG}$ ). B, sagittal slices corresponding to dashed lines in A. C, PET (MIPs) of patient \#3 (f, $37 \mathrm{y}, 61 \mathrm{~kg}$; $286 \mathrm{MBq}\left[{ }^{18} \mathrm{~F}\right] \mathrm{FDG} 71 \mathrm{~min}$ p.i.; $135 \mathrm{MBq}{ }^{68} \mathrm{Ga}$-Trivehexin, $A_{\mathrm{M}}=29 \mathrm{MBq} / \mathrm{nmol}$, total peptide amount $4.7 \mathrm{nmol}$, $67 \mathrm{~min}$ p.i.) suffering from a solitary prelaryngeal cutaneous metastasis of a previously resected and irradiated left-sided parotideal adenocarcinoma. D, Axial slices corresponding to dashed lines in C. SUV $V_{\max }$ of the metastasis was 6.1 for $\left[{ }^{18} \mathrm{~F}\right] \mathrm{FDG}$ and 7.6 for ${ }^{68} \mathrm{Ga}$-Trivehexin, metabolic tumor volume was $2.2 \mathrm{~cm}^{3}$. E, $\beta 6$-integrin IHC of excised tissue of the patient shown in $\mathbf{C}+\mathbf{D}$; magnifications are shown for the central tumor area (orange box), the infiltrative margin (cyan box), and non-tumor tissue (violet box). Bars represent $1 \mathrm{~mm}$ for overview and $100 \mu \mathrm{m}$ for the magnifications, respectively. 

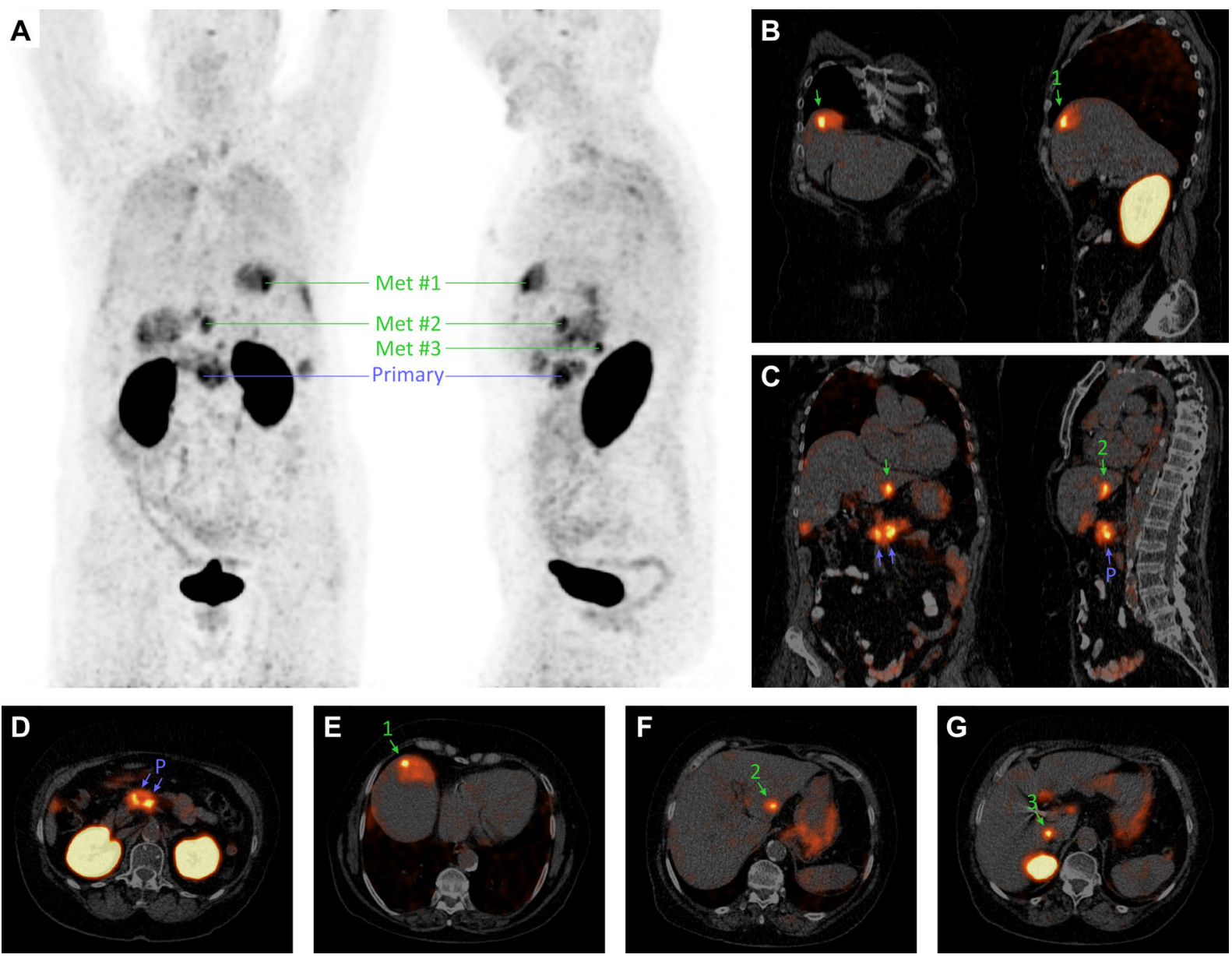

Figure 5: ${ }^{68} \mathrm{Ga}$-Trivehexin PET/CT of metastatic PDAC (confirmed by biopsy) in patient \#4 (f, 80 y, $80 \mathrm{~kg}, 105 \mathrm{MBq}$, 120 min p.i.). PET is scaled to SUV 10 in all images. A, anterior and sagittal MIP, showing ${ }^{68}$ Ga-Trivehexin uptake in the primary tumor ( $\left.S U V_{\max }=9.8-10.9\right)$ and in 7 metastases. Selected metastases highlighted in panels B-G are indicated $\left(\mathrm{SUV}_{\max }\right.$ of \#1, \#2, and \#3 are 10.9, 10.0, and 9.9, respectively). B, coronal and sagittal PET/CT slices through metastasis \#1. C, coronal and sagittal PET/CT slices through primaries and metastasis \#2. D, E, F, G, axial $\mathrm{PET} / \mathrm{CT}$ slices though primaries and metastases \#1, \#2, and \#3, respectively.

\section{Discussion}

\section{Strategy for optimization of biokinetics}

In order to exploit the multimer effect to achieve a higher target affinity, enhanced uptake, and longer retention [41], we reasoned that increasing the intrinsic hydrophilicity of c[FRGDLAFp $(N \mathrm{Me}) \mathrm{K}$ ] should both accelerate blood clearance and reduce non-specific uptake of the corresponding trimers. An exchange of both phenylalanines by tyrosines appeared to be a convenient and simple strategy, yet at the risk of considerably lowering target affinity and selectivity which, fortunately, did not occur. $\alpha \mathrm{v} \beta 6$ integrin affinities of ${ }^{68} \mathrm{Ga}-\mathrm{TRAP}(\mathrm{AvB} 6)_{3}[17]$ and ${ }^{68} \mathrm{Ga}$-Trivehexin were in the same, low double-digit picomolar range (23 and $33 \mathrm{pM}$, respectively), and ${ }^{68} \mathrm{Ga}$-Trivehexin showed a remarkable selectivity for $\alpha v \beta 6$ over other RGD-binding integrins.

Of note, the difference between both cyclic peptide monomers is minimal on an atomic level. $\mathrm{c}[\mathrm{YRGDLAYp}(N \mathrm{Me}) \mathrm{K}]$ merely contains two additional oxygens (see Figure 1), which means that the 
trimer ${ }^{68} \mathrm{Ga}$-Trivehexin incorporates just 6 oxygen atoms more than ${ }^{68} \mathrm{Ga}$-TRAP(AvB6) 3 , the gain in molecular weight amounting to only $96 \mathrm{Da}$. This is comparable to the mass of 2 polyethylene glycol (PEG) units ( $\left.\left(-\mathrm{CH}_{2}-\mathrm{CH}_{2}-\mathrm{O}-\right)_{2}, 88 \mathrm{Da}\right)$, or approximately half of a monoglycosyl moiety $\left(\mathrm{C}_{6} \mathrm{H}_{12} \mathrm{O}_{6}\right.$, $180 \mathrm{Da}$ ), which has been attached as a polar modifier to RGD peptides before [46]. To our delight, ${ }^{68} \mathrm{Ga}-$ Trivehexin nonetheless exhibited dramatically improved biokinetics (see Figure 2), above all, a rapid clearance from the blood pool, despite its rather modest increase in hydrophilicity $\left(\Delta \log D_{7.4}=-0.6\right)$. With exception of the kidneys, non-specific uptakes were virtually completely eliminated, which is most appreciated for liver and lung because these organs are typical locations of primaries and metastases of av $\beta 6$-integrin expressing carcinomas. The development of ${ }^{68} \mathrm{Ga}$-Trivehexin showcases that it can be much more rewarding to carefully evaluate any possibility to optimize pharmacokinetics of radiopharmaceuticals by structural alteration of the respective bioligand itself, rather than attempting to mitigate unfavorable pharmacokinetics by PEGylation [17,47] or other pharmacokinetic modifiers [46]. The comparison of ${ }^{68} \mathrm{Ga}$-TRAP(AvB6) 3 and ${ }^{68} \mathrm{Ga}$-Trivehexin furthermore exemplifies that a successful multimerization might not in all cases be a strictly deterministic result of a thoughtful choice of scaffolds, linkers, symmetry, degeneracy coefficients, or other factors [41]. Multimers should rather be considered complex systems and their properties be perceived as emergent phenomena [48], leading to the simple truth that some receptor ligands are apparently suitable for multimerization whereas others, even those with an almost identical chemical structure and similar properties, are not [49]. Neither do we believe that the dramatically improved pharmacokinetics of ${ }^{68} \mathrm{Ga}$-Trivehexin could have been predicted from any in-vitro parameter, such as its affinity, selectivity, or its only moderately improved hydrophilicity. We therefore like to emphasize that the characteristics of more complex molecular systems, such as multimers, are to a considerable extent a consequence of their higher integrative level and thus, by principle, can neither be fully predicted on the basis of a few measured or calculated parameters, nor concluded upon from the features of their constituents [48].

\section{Possible Clinical Implications of ${ }^{68} \mathrm{Ga}$-Trivehexin PET}

The preclinical and in-human PET results are largely in accordance. No relevant non-specific uptake was observed in any organ, except in the kidneys. Similar to the previously reported $\alpha v \beta 8$-integrin tracer ${ }^{68} \mathrm{Ga}$-Triveoctin, the kidney uptake observed in humans (SUV-based kidney-to-blood ratio of $\approx 29$, 97 min p.i.) was lower than in mice (kidney-to-blood ratio of $\approx 93,90 \mathrm{~min}$ p.i.). Overall, the presented preclinical and first-in-human data strongly suggest that ${ }^{68} \mathrm{Ga}$-Trivehexin is indeed a suitable agent for non-invasive mapping of $\alpha v \beta 6$-integrin expression in a clinical setting. The presented work is limited by the fact that only single cases have been examined, obviously not allowing for more than an educated guess on the future clinical impact of ${ }^{68} \mathrm{Ga}$-Trivehexin PET. We nonetheless assume that the clinical scope encompasses all purposes that have been suggested for other $\alpha v \beta 6$-integrin targeted radiopharmaceuticals before $[17,21,25,26,27,28,29,30,31,32]$. 
We particularly envisage an added value of ${ }^{68} \mathrm{Ga}$-Trivehexin over $\left[{ }^{18} \mathrm{~F}\right] \mathrm{FDG}$ in cases where the latter shows a low sensitivity or specificity. Figure 4 demonstrates that ${ }^{68}$ Ga-Trivehexin PET might allow for an improved delineation of head-and-neck cancers because it apparently shows a lower uptake in lymphatic tissue and tumor-associated inflammation. Such information might be valuable for planning external beam therapy regimen. In the same context, ${ }^{68} \mathrm{Ga}$-Trivehexin PET might enable an improved follow-up because it could facilitate the assessment of an early response to radiation therapy, which sometimes is not easily possible with $\left[{ }^{18} \mathrm{~F}\right] \mathrm{FDG}$ due to its pronounced uptake in tissues afflicted by radiogenic inflammation. ${ }^{68} \mathrm{Ga}$-Trivehexin might furthermore enable a more specific and sensitive diagnosis of metastatic carcinomas, particularly those which are characterized by a low metabolic conversion rate and/or a high fraction of stroma. Among those, pancreatic carcinoma is arguably the most important target, because it is one of the cancers with the worst overall prognosis and $\left[{ }^{18} \mathrm{~F}\right] \mathrm{FDG}$ possesses only a limited value for early detection of PDAC [50]. Figure 5 admittedly does not allow for more than a first glimpse on ${ }^{68} \mathrm{Ga}$-Trivehexin driven PET diagnostics of metastatic PDAC. We nevertheless hold the view that the demonstrated feasibility of detecting PDAC primaries and even small metastases might turn out to be a game changer, all the more because it also implicates the possibility of a differential diagnosis of PDAC vs. pancreatitis, or even the detection of $\alpha v \beta 6$-integrin expressing PDAC precursor lesions, pancreatic intraepithelial neoplasias (PanIN) [21]. Although not supported by the currently available data, we furthermore imagine that ${ }^{68} \mathrm{Ga}$-Trivehexin might turn out to be a suitable agent for monitoring the response to established PDAC chemotherapies like folfirinox and, as such, might claim a position in regular PDAC treatment regimen. Such applications, however, obviously need to be firmly established in future clinical trials. The same applies to other potential uses, e.g., imaging of fibrosis [29] or monitoring of the response to anti-fibrotic inhalation therapy with $\alpha v \beta 6$-integrin antagonists [31,32].

\section{Conclusion}

Trimerization of an $\alpha v \beta 6$-integrin binding cyclic nonapeptide on the TRAP chelator core resulted in the novel PET probe ${ }^{68}$ Ga-Trivehexin, which showed an exceptional $\alpha v \beta 6$-integrin affinity of $33 \mathrm{pM}$, a high selectivity over other RGD-binding integrin subtypes, and enabled sensitive and specific imaging of $\alpha v \beta 6$-integrin expression in murine tumor xenografts. The preclinical data corresponded remarkably well to first clinical PET/CT in cancer patients. ${ }^{68} \mathrm{Ga}$-Trivehexin showed a high and persisting uptake in HNSCC ( $\left.\mathrm{SUV}_{\max }=13.1\right)$ as well as in primaries and liver metastases of PDAC (SUV $\max _{\text {about }}$ 10). No relevant uptakes were seen in other organs and tissues, except excretion-related in the kidneys and urinary tract which did not compromise tumor visualization in the investigated settings. We conclude that ${ }^{68} \mathrm{Ga}$-Trivehexin possesses a high clinical value for PET imaging of all indications and conditions known to be frequently associated with elevated $\alpha v \beta 6$-integrin expression, such as various carcinomas (pancreatic adeno, head-and-neck squamous, colorectal, cervical, lung adeno, and others), as well as fibrosis. 


\section{Declarations}

\section{Funding}

This work was funded by the Deutsche Forschungsgemeinschaft (SFB 824, projects A10 and Z2).

\section{Competing interests}

N.G.Q., K.S., and J.N. are co-inventors of patents related to ${ }^{68} \mathrm{Ga}$-Trivehexin. J.N. is shareholder of TRIMT GmbH (Radeberg, Germany), which is active in the field of radiopharmaceutical develeopment.

\section{Ethics approval}

Procedures involving laboratory mice and their care were conducted in conformity with institutional guidelines and with approval from the responsible local authorities (Regierung von Oberbayern, ROB55.2-2532.Vet_2-18-109).

\section{Consent to participate}

The authors affirm that the patients provided written informed consent prior to the investigations.

\section{Consent for publication}

The authors affirm that the patients provided written informed consent for publication of data and images.

\section{Availability of data and materials}

The datasets used and/or analysed during the current study are available from the corresponding author on reasonable request.

\section{Authors' contributions}

Conceived and designed the experiment: NGQ, KS, NC, CP, JK, JN. Performed the experiments: NGQ, NC, MAZ, MP, FR, SH, JK. Analyzed the data: NGQ, SH, SK, WW, JK, JN. Wrote the original manuscript: JN. All authors approved the final version of the manuscript. 


\section{Acknowledgments}

We thank Marion Mielke and Annett Hering (CEP, Institute of Pathology, TUM) for excellent technical support, Prof. Korinna Jöhrens (Institute of Pathology, University Hospital Carl Gustav Carus, Dresden) for excellent cooperation, and Markus Mittelhäuser and Hannes Rolbieski for assistance with animal experiments.

\section{References}

1 Sontag S. Illness as metaphor. Farrar, Straus \& Giroux, New York, 1978, ISBN: 978-0-374-17443-9.

2 Mukherjee S. The Emperor of All Maladies: A Biography of Cancer. Scribner, New York, 2010, ISBN: 9781-4391-0795-9.

3 Virchow R. Die krankhaften Geschwülste. August Hirschwald, Berlin, 1863.

4 Brooks PC, Clark RAF, Cheresh DA. Requirement Of Vascular Integrin $\alpha_{v} \beta_{3}$ For Angiogenesis. Science. 1994;264:569-71.

5 Avraamides CJ, Garmy-Susini B, Varner JA. Integrins in angiogenesis and lymphangiogenesis. Nat Rev Cancer. 2008;8:604-17.

6 Hamidi H, Ivaska J. Every step of the way: integrins in cancer progression and metastasis. Nat Rev Cancer. 2018;18:533-48.

7 Nieberler M, Reuning U, Reichart F, Notni J, Wester HJ, Schwaiger M, et al. Exploring the Role of RGDRecognizing Integrins in Cancer. Cancers. 2017;9:116.

8 Hanahan D, Weinberg RA. The hallmarks of cancer. Cell. 2000;100:57-70.

9 Brown NF, Marshall JF. Integrin-Mediated TGF $\beta$ Activation Modulates the Tumour Microenvironment. Cancers. 2019;11:1221.

10 Blobe GC, Schiemann WP, Lodish HF. Role of transforming growth factor $\beta$ in human disease. N Engl J Med. 2000;34:1350-8.

11 Dong X, Zhao B, Iacob RE, Zhu J, Koksal AC, Lu C, et al. Force interacts with macromolecular structure in activation of TGF- $\beta$. Nature. 2017;542:55-9.

12 Ha T. Growth factor rattled out of its cage. Nature. 2017;542:40-1.

13 Worthington JJ, Klementowicz JE, Travis MA. TGF $\beta$ : a sleeping giant awoken by integrins. Trends Biochem Sci. 2011;36:47-54.

14 Adorno M, Cordenonsi M, Montagner M, Dupont S, Wong C, Hann B, et al. A Mutant-p53/Smad Complex Opposes p63 to Empower TGFß-Induced Metastasis. Cell. 2009;137:87-98.

15 Ahmed S, Bradshaw AD, Geta S, Dewan MZ, Xu R. The TGF $\beta /$ Smad4 signalling pathway in pancreatic carcinogenesis and its clinical significance. J Clin Med. 2017;6:5-15. 
16 Inman GJ. Switching TGF $\beta$ from a tumor suppressor to a tumor promoter. Curr Opin Genet Dev. 2011;21:9399.

17 Notni J, Reich D, Maltsev OV, Kapp TG, Steiger K, Hoffmann F, et al. In-vivo PET Imaging of the Cancer Integrin $\alpha v \beta 6$ Using ${ }^{68} \mathrm{Ga}$-Labeled Cyclic RGD Nonapeptides. J Nucl Med. 2017;58:671-7.

18 Koopman Van Aarsen LA, Leone DR, Ho S, Dolinski BM, McCoon PE, LePage DJ, et al. AntibodyMediated Blockade of Integrin $\alpha_{v} \beta_{6}$ Inhibits Tumor Progression In vivo by a Transforming Growth Factor$\beta-$ Regulated Mechanism. Cancer Res. 2008;68:561-70.

19 Sipos B, Hahn D, Carceller A, Piulats J, Hedderich J, Kalthoff H, et al. Immunohistochemical screening for $\beta_{6}$-integrin subunit expression in adenocarcinomas using a novel monoclonal antibody reveals strong upregulation in pancreatic ductal adenocarcinomas in vivo and in vitro. Histopathol. 2004;45:226-36.

20 Reader CS, Vallath S, Steele CW, Haider S, Brentnall A, Desai A, et al. The integrin $\alpha v \beta 6$ drives pancreatic cancer through diverse mechanisms and represents an effective target for therapy. J Pathol. 2019;249:33242.

21 Steiger K, Schlitter AM, Weichert W, Esposito I, Wester HJ, Notni J. Perspective of avß6-Integrin Imaging for Clinical Management of Pancreatic Carcinoma and Its Precursor Lesions. Mol Imaging. 2017; 16:1536012117709384.

22 Niu J, Li Z. The roles of integrin $\alpha v \beta 6$ in cancer. Cancer Lett. 2017;403:128e137.

23 Tummers WS, Willmann JK, Bonsing BA, Vahrmeijer AL, Gambhir SS, Swijnenburg RJ. Advances in Diagnostic and Intraoperative Molecular Imaging of Pancreatic Cancer. Pancreas. 2018;47:675-89.

24 Horan GS, Wood S, Ona V, Li DJ, Lukashev ME, Weinreb PH et al. Partial inhibition of integrin av $\beta 6$ prevents pulmonary fibrosis without exacerbating inflammation. Am J Respir Crit Care Med. 2008;177:5665.

25 Altmann A, Sauter M, Roesch S, Mier W, Warta R, Debus J, et al. Identification of a Novel ITG $\alpha_{v} \beta_{6}$-Binding Peptide Using Protein Separation and Phage Display. Clin Cancer Res. 2017;23:4170-80.

26 Roesch S, Lindner T, Sauter M, Loktev A, Flechsig P, Müller M, et al. Comparison of the RGD MotifContaining $\alpha_{v} \beta_{6}$ Integrin-Binding Peptides SFLAP3 and SFITGv6 for Diagnostic Application in HNSCC. J Nucl Med. 2018;59:1679-85.

27 Müller M, Altmann A, Sauter M, Lindner T, Jäger D, Rathke H, et al. Preclinical evaluation of peptide-based radiotracers for integrin $\alpha v \beta 6$-positive pancreatic carcinoma. Nuklearmedizin. 2019;58:309-18.

28 Flechsig P, Lindner T, Loktev A, Roesch S, Mier W, Sauter M, et al. PET/CT Imaging of NSCLC with a $\alpha_{v} \beta_{6}$ Integrin-Targeting Peptide. Mol Imaging Biol. 2019;21:973-83.

29 Kimura RH, Wang L, Shen B, Huo L, Tummers W, Filipp FV, et al. Evaluation of integrin $\alpha_{v} \beta_{6}$ cystine knot PET tracers to detect cancer and idiopathic pulmonary fibrosis. Nat Commun. 2019;10,4673.

30 Hausner SH, Bold RJ, Cheuy LY, Chew HK, Daly ME, Davis RA, et al. Preclinical Development and Firstin-Human Imaging of the Integrin $\alpha v \beta 6$ with $\left[{ }^{18} \mathrm{~F}\right] \alpha_{v} \beta_{6}$-Binding Peptide in Metastatic Carcinoma. Clin Cancer Res. 2019;25:1206-15. 
31 Lukey PT, Coello C, Gunn R, Parker C, Wilson FJ, Saleem A, et al. Clinical quantification of the integrin $\alpha v \beta 6$ by $\left[{ }^{18} \mathrm{~F}\right] \mathrm{FB}-\mathrm{A} 20 \mathrm{FMDV} 2$ positron emission tomography in healthy and fibrotic human lung (PETAL Study). Eur J Nucl Med Mol Imaging. 2020;47:967-79.

32 Maher TM, Simpson JK, Porter JC, Wilson FJ, Chan R, Eames R, et al. A positron emission tomography imaging study to confirm target engagement in the lungs of patients with idiopathic pulmonary fibrosis following a single dose of a novel inhaled $\alpha v \beta 6$ integrin inhibitor. Respirat Res. 2020;21:75.

33 Notni J, Šimeček J, Hermann P, Wester HJ. TRAP, a powerful and versatile framework for gallium-68 radiopharmaceuticals. Chemistry. 2011;17:14718-22.

34 Maltsev OV, Marelli UK, Kapp TG, Di Leva FS, Di Maro S, Nieberler M, et al. Stable Peptides Instead of Stapled Peptides: Highly Potent $\alpha_{v} \beta_{6}$-Selective Integrin Ligands. Angew Chem Int Edit. 2016;55:1535-9.

35 Färber SF, Wurzer A, Reichart F, Beck R, Kessler H, Wester HJ, et al. Therapeutic Radiopharmaceuticals Targeting Integrin $\alpha v \beta 6$. ACS Omega. 2018;3:2428-36.

36 Notni J, Pohle K, Wester HJ. Be spoilt for choice with radiolabeled RGD peptides: Preclinical evaluation of ${ }^{68} \mathrm{Ga}$-TRAP(RGD)3. Nucl Med Biol. 2013;40:33-41.

37 Notni J, Steiger K, Hoffmann F, Reich D, Kapp TG, Rechenmacher F, et al. Complementary, Selective PETImaging of Integrin Subtypes $\alpha_{5} \beta_{1}$ and $\alpha_{v} \beta_{3}$ Using Ga-68-Aquibeprin and Ga-68-Avebetrin. J Nucl Med. 2016;57:460-6.

38 Kapp TG, Di Leva FS, Notni J, Räder AFB, Fottner M, Reichart F, et al. $N$-Methylation of isoDGR Peptides: Discovery of a Selective $\alpha 5 \beta 1$-Integrin Ligand as a Potent Tumor Imaging Agent. J Med Chem. 2018;61:2490-9

39 Quigley NG, Tomassi S, Di Leva FS, Di Maro S, Richter F, Steiger K, et al. Click-chemistry (CuAAC) trimerization of an $\alpha v \beta 6$-integrin targeting Ga-68-peptide: Enhanced contrast for in-vivo PET imaging of human lung adenocarcinoma xenografts. ChemBioChem. 2020;21:2836-43.

40 Quigley NG, Steiger K, Richter F, Weichert W, Hoberück S, Kotzerke J, et al. Tracking a TGF- $\beta$ activator in vivo: sensitive PET imaging of $\alpha v \beta 8$-integrin with the Ga-68-labeled cyclic RGD octapeptide trimer Ga68-Triveoctin. EJNMMI Res. 2020;10:133.

41 Böhmer VI, Szymanski V, Feringa BL, Elsinga PH. Multivalent Probes in Molecular Imaging: Reality or Future? Trends Mol Med. 2021;27:379-93.

42 Kapp TG, Rechenmacher F, Neubauer S, Notni J, Räder AFB, Fottner M, et al. A Comprehensive Evaluation of the Activity and Selectivity Profile of Ligands for RGD-binding Integrins. Sci Rep. 2017;7:39805.

43 Notni J, Šimeček J, Wester HJ. Phosphinic acid functionalized polyazacycloalkane chelators for radiodiagnostics and radiotherapeutics: unique characteristics and applications. ChemMedChem. 2014;9:1107-15.

44 Notni J, Hermann P, Havlíčková J, Kotek J, Kubíček V, Plutnar J, et al. A triazacyclononane-based bifunctional phosphinate ligand for the preparation of multimeric $68 \mathrm{Ga}$ tracers for positron emission tomography. Chem Eur J. 2010;16:7174-85. 
45 Notni J, Wester HJ. A Practical Guide on the Synthesis of Metal Chelates for Molecular Imaging and Therapy by Means of Click Chemistry. Chem Eur J. 2016;22:11500-8.

46 Haubner R, Wester HJ, Burkhart F, Senekowitsch-Schmidtke R, Weber W, Goodman SL, et al. Glycosylated RGD-Containing Peptides: Tracer for Tumor Targeting and Angiogenesis Imaging with Improved Biokinetics. J Nucl Med. 2001;42:326-36.

47 Hausner SH, Bauer N, Hu LY, Knight LM, Sutcliffe JL. The Effect of Bi-Terminal PEGylation of an Integrinavß6 Targeted ${ }^{18} \mathrm{~F}$ Peptide on Pharmacokinetics and Tumor Uptake. J Nucl Med. 2015;56:784-90.

48 Corning PA. The re-emergence of "emergence": A venerable concept in search of a theory. Complexity. 2002;7:18-30.

49 Maschauer S, Einsiedel J, Reich D, Hübner H, Gmeiner P, Wester HJ, et al. Theranostic Value of Multimers: Lessons Learned from Trimerization of Neurotensin Receptor Ligands and Other Targeting Vectors. Pharmaceuticals. 2017;10:29.

50 Strobel O, Büchler MW. Pancreatic cancer: FDG-PET is not useful in early pancreatic cancer diagnosis. Nat Rev Gastroenterol Hepatol. 2013;4:203-5. 


\section{Supplementary Files}

This is a list of supplementary files associated with this preprint. Click to download.

- trivehexinsoloSIV1.pdf 